01

\title{
К работе Г.Б. Малыкина и В.И. Поздняковой «К вопросу о скорости движения светового „зайчика“ по экрану цилиндрической формы»
}

\author{
(C) Н.В. Купряев
}

Физический институт им. П.Н. Лебедева РАН, Самарский филиал, 443011 Самара, Россия

e-mail: kuprjaev@front.ru

(Поступило в Редакцию 5 декабря 2016 г.)

Показано, что в работе Г.Б. Малыкина и В.И. Поздняковой «К вопросу о скорости движения светового „зайчика“ по экрану цилиндрической формы», опубликованной в первом выпуске ЖТФ за 2007 год, допущены неточности при расчете скорости движения светового „зайчика“ по экрану цилиндрической формы. В результате получены неправильные формулы расчета скорости движения светового „зайчика“ применительно к классической механике и к преобразованию Тангерлини. Приведены правильные формулы расчета скорости движения светового „зайчика“ по экрану цилиндрической формы применительно к классической механике и к преобразованию Тангерлини.

DOI: 10.21883/JTF.2017.07.44665.2127

В первом выпуске ЖТФ за 2007 г. [1] была опубликована работа Г.Б. Малыкина и В.И. Поздняковой «К вопросу о скорости движения светового „зайчика“ по экрану цилиндрической формы», в которой предлагался метод проверки анизотропии скорости света с помощью светового „зайчика“, движущегося со сверхсветовой скоростью по экрану цилиндрической формы.

По мнению авторов [1], если имеет место анизотропия скорости света в движущейся инерциальной системе отсчета (ИСО) $S^{\prime}$, должна меняться скорость движения светового „зайчика“ по экрану цилиндрической формы в зависимости от угла, под которым направлен свет от вращающегося прожектора.

К сожалению, в работе при расчете скорости движения светового „зайчика“ в ИСО $S^{\prime}$ были допущены неточности. Так применительно к классической механике $(V \ll c)$ при расчете скорости движения ,зайчика“ по экрану цилиндрической формы вместо формулы скорости света в ИСО $S^{\prime}$ (см. Приложение в конце работы)

$$
c^{\prime}=c\left(\sqrt{1-\frac{V^{2}}{c^{2}} \sin ^{2} \theta^{\prime}}-\frac{V}{c} \cos \theta^{\prime}\right),
$$

где $V$ - скорость ИСО $S^{\prime}$ относительно эфира (ИСО $S$ ). Эта формула, выраженная через скорость $v_{\text {aeth }}^{\prime}$ эфира относительно ИСО $S^{\prime}$ применительно к ситуации [1], имеет вид

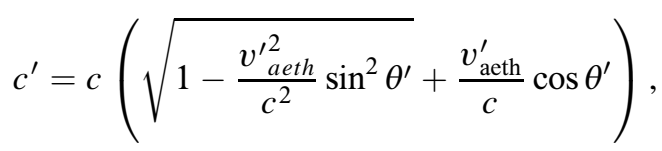

где $\theta^{\prime}-$ угол, под которым распространяется свет в ИСО $S^{\prime}$ и под которым направлен прожектор. Была ошибочно использована формула

$$
c^{\prime}=c \sqrt{1+2 \frac{v_{\mathrm{aeth}}^{\prime}}{c} \cos \theta+\frac{v_{\mathrm{aeth}}^{\prime 2}}{c^{2}}},
$$

где $\theta-$ угол, под которым распространяется свет в ИСО $S$, но не в ИСО $S^{\prime}$. Формула (3), выраженная через скорость $V$ ИСО $S^{\prime}$, имеет вид

$$
c^{\prime}=c \sqrt{1-2 \frac{V}{c} \cos \theta+\frac{V^{2}}{c^{2}}}
$$

В результате для скорости $v_{\text {proj }}^{\prime}$ движения светового „зайчика“ по экрану цилиндрической формы в ИСО $S^{\prime}$ применительно к классической механике $\left(v_{\text {aeth }}^{\prime} \ll c\right)$ была ошибочно получена формула

$$
\begin{aligned}
& v_{\text {proj }}^{\prime}(t)=R^{\prime} \Omega \\
& \times \frac{\left(1+\frac{v_{\text {acth }}^{\prime}}{c} \cos \Omega t\right) \sqrt{1+2 \frac{v_{\text {acth }}^{\prime}}{c} \cos \Omega t+\frac{v_{\text {acth }}^{\prime 2}}{c^{2}}}}{\left(1+2 \frac{v_{\text {aeth }}^{\prime}}{c} \cos \Omega t+\frac{v_{\text {aeth }}^{\prime 2}}{c^{2}}\right)^{3 / 2}+R^{\prime} \Omega \frac{v_{\text {aeth }}^{\prime}}{c^{2}} \sin \Omega t},
\end{aligned}
$$

где $\Omega t=\theta-$ угол, под которым распространяется свет в ИСО $S$, но не в ИСО $S^{\prime}$, в момент времени $t$, $\Omega$ - угловая скорость вращения прожектора в ИСО $S$, $R^{\prime}$ - расстояние от источника излучения до экрана в ИСО $S^{\prime}$, которая не совсем правильно описывает движения ,зайчика“.

В действительности формула (3) или, что то же самое, формула (4) представляет собой не скорость света в ИСО $S^{\prime}$ (см. Приложение в конце работы), а лишь преобразование скорости света при переходе из ИСО $S$ в ИСО $S^{\prime}$.

В самой ИСО $S^{\prime}$ скорость света применительно к классической механике определяется не формулой (3), а формулой (1) (см. Приложение в конце работы), 
или, что то же самое, формулой (2), если $V$ выразить через $v_{\text {aeth. }}^{\prime}$ В результате для скорости $v_{\text {proj }}^{\prime}$ движения светового „зайчика“ по экрану цилиндрической формы в ИСО $S^{\prime}$ применительно к классической механике $(V \ll c)$ получается не формула $(5)$, а формула

$$
v_{\text {proj }}^{\prime}\left(t^{\prime}\right)=\frac{R^{\prime} \Omega^{\prime}}{1-R^{\prime} \Omega^{\prime} \frac{V}{c^{2}} \frac{\cos \Omega^{\prime} t^{\prime}}{\sqrt{1-\frac{V^{2}}{c^{2}} \sin ^{2} \Omega^{\prime} t^{\prime}}}},
$$

где $\Omega^{\prime} t^{\prime}=\theta^{\prime}$ - угол, под которым распространяется свет в ИСО $S^{\prime}$ и под которым направлен прожектор в ИСО $S^{\prime}$ в момент излучения $t^{\prime}$ света; $\Omega^{\prime}-$ угловая скорость вращения прожектора в ИСО $S^{\prime}$. Эта формула в отличие от (5) правильно описывает движения „зайчика“ в классической механике.

Применительно же к преобразованию Тангерлини [2] авторы [1] формулу для скорости света $c^{\prime}$ в ИСО $S^{\prime}$ практически правильно написали, но не совсем точно. Вместо правильной формулы

$$
c^{\prime}=\frac{c}{1-\frac{v_{\text {aeth }}^{\prime}}{c}\left(1-\frac{V^{2}}{c^{2}}\right) \cos \theta^{\prime}}
$$

(эта формула, если $v_{\text {aeth }}^{\prime}$ выразить через скорость $V$ ИСО $S^{\prime}$ относительно эфира $($ ИСО $S)$ ) имеет вид

$$
c^{\prime}=\frac{c}{1+\frac{V}{c} \cos \theta^{\prime}},
$$

было ошибочно записано

$$
c^{\prime}=\frac{c}{1-\frac{v_{\mathrm{aeth}}^{\prime}}{c} \cos \theta^{\prime}} .
$$

И в результате для скорости $v_{\text {рroj движения свето- }}^{\prime}$ вого „зайчика“ по экрану цилиндрической формы в ИСО $S^{\prime}$ применительно к преобразованию Тангерлини была ошибочно получена формула

$$
v_{\text {proj }}^{\prime}\left(t^{\prime}\right)=\frac{R^{\prime} \Omega^{\prime}}{1+R^{\prime} \Omega^{\prime} \frac{v_{\text {aeth }}^{\prime}}{c^{2}} \sin \Omega^{\prime} t^{\prime}},
$$

которая не совсем правильно описывает поведение движения „зайчика“ по экрану цилиндрической формы применительно к преобразованию Тангерлини.

В действительности получается формула

$$
v_{\text {proj }}^{\prime}\left(t^{\prime}\right)=\frac{R^{\prime} \Omega^{\prime}}{1+R^{\prime} \Omega^{\prime} \frac{v_{\text {aeth }}^{\prime}}{c^{2}}\left(1-\frac{V^{2}}{c^{2}}\right) \sin \Omega^{\prime} t^{\prime}}
$$

или, что то же самое, если $v_{\text {aeth }}^{\prime}$ выразить через скорость $V$ ИСО $S^{\prime}$ относительно эфира $($ ИСО $S$ )

$$
v_{\text {proj }}^{\prime}\left(t^{\prime}\right)=\frac{R^{\prime} \Omega^{\prime}}{1-R^{\prime} \Omega^{\prime} \frac{V}{c^{2}} \sin \Omega^{\prime} t^{\prime}},
$$

которая правильно описывает поведение движения „зайчика“ по экрану цилиндрической формы в ИСО $S^{\prime}$ в рамках преобразования Тангерлини.

Зависимости $v_{\text {proj }}^{\prime}\left(t^{\prime}\right)$, рассчитанные с помощью формул (12) и (6), практически не различаются и при $V \ll c$ совпадают. Кроме того, формулы (12) и (6) по форме похожи друг на друга и различаются лишь коэффициентами при $\sin \Omega^{\prime} t^{\prime}$. Формула (12) является при этом точной, т.е. при больших $V$, а формула (6) приближенной, т. е. при $V \ll c$.

Формулы же (10) и (5), полученные авторами [1], даже по форме сильно различаются. Поэтому даже по одному этому признаку можно было догадаться, что формула (5) является ошибочной.

Поэтому в действительности, если имеет место анизотропия скорости света в движущейся ИСО $S^{\prime}$, поведение движения „зайчика“ по экрану цилиндрической формы в ИСО $S^{\prime}$ описывается не формулами (5) или (10), а формулами (6) при $V \ll c$ или (12) при больших $V$.

\section{Приложение}

То, что в ИСО $S^{\prime}$ скорость $c^{\prime}$ света применительно к классической механике определяется именно формулой (1) или, что то же самое, формулой (2), а не (3), легко доказать. Действительно, в классической механике скорости $\left(v_{x}, v_{y}, v_{z}\right)$ и $\left(v_{x}^{\prime}, v_{y}^{\prime}, v_{z}^{\prime}\right)$ в ИСО $S$ и $S^{\prime}$ связаны преобразованием

$$
v_{x}^{\prime}=v_{x}-V, \quad v_{y}^{\prime}=v_{y}, \quad v_{z}^{\prime}=v_{z} .
$$

Записывая преобразование (13) в виде

$$
v^{\prime} \equiv \sqrt{v_{x}^{\prime 2}+v_{y}^{\prime 2}+v_{z}^{2}}=\sqrt{v^{2}-2 v_{x} V+V^{2}}
$$

и введя обозначения $v^{\prime}=c^{\prime}, v=c, v_{x}=c \cos \theta$, получаем формулу преобразования скорости света при переходе из ИСО $S$ в ИСО $S^{\prime}$ :

$$
c^{\prime}=c \sqrt{1-2 \frac{V}{c} \cos \theta+\frac{V^{2}}{c^{2}}},
$$

которая совпадает с (4).

Чтобы найти теперь скорость $c^{\prime}$ света в самой ИСО $S^{\prime}$, т.е. выраженной через угол $\theta^{\prime}$, под которым распространяется свет в ИСО $S^{\prime}$ и под которым направлен прожектор, угол $\theta$ в формуле (4) нужно преобразовать. Для этого нужно, например, переписать преобразование компоненты $v_{x}$ в преобразовании (13) в виде

$$
c^{\prime} \cos \theta^{\prime}=c \cos \theta-V
$$

найти отсюда

$$
\cos \theta=\frac{c^{\prime} \cos \theta^{\prime}+V}{c}
$$

и подставить полученное выражение в преобразование скорости света (4). В результате получится

$$
c^{\prime}=\sqrt{c^{2}-2 V c^{\prime} \cos \theta^{\prime}-V^{2}} \text {. }
$$


Решая уравнение относительно $c^{\prime}$, и получаем в итоге искомую скорость света в ИСО $S^{\prime}$

$$
c^{\prime}=c\left(\sqrt{1-\frac{V^{2}}{c^{2}} \sin ^{2} \theta^{\prime}}-\frac{V}{c} \cos \theta^{\prime}\right),
$$

которая совпадает с (1).

Это и есть, таким образом, скорость света $c^{\prime}$ в ИСО $S^{\prime}$ применительно к классической механике. Очевидно, что направление распространения света в ИСО $S^{\prime}$ (угол $\theta^{\prime}$ ) совпадает с направлением прожектора. Действительно, предположим, что в начале координат ИСО $S^{\prime}$ в точке $O^{\prime}$ (см. рисунок) находится неподвижно точечный источник световых волн и пусть на некотором расстоянии от начала координат вдоль оси $y^{\prime}$ находится перпендикулярно оси $y^{\prime}$ экран с отверстием. Это модель прожектора. Прожектор направлен вдоль оси $y^{\prime}$. ИСО $S^{\prime}$, очевидно, движется относительно ИСО $S$ (эфира) со скоростью $V$ вдоль положительной оси $x$.

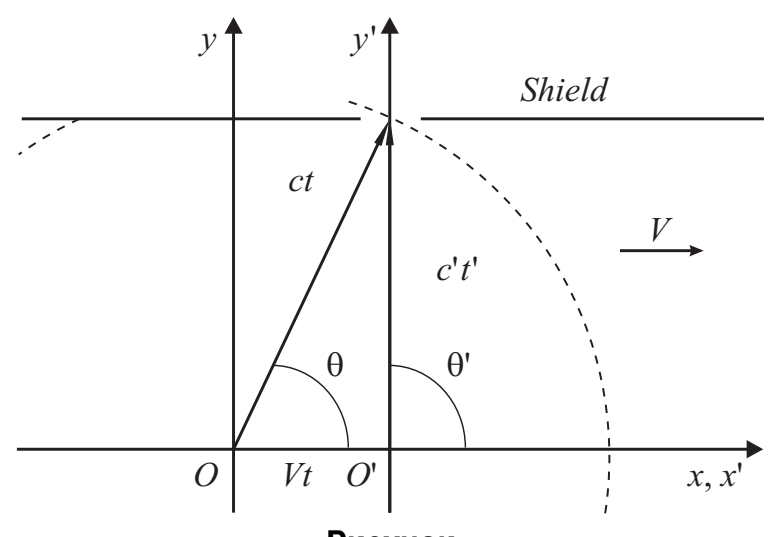

Рисунок

Пусть в момент времени $t^{\prime}=t=0$ источник излучает сферическую волну (штриховая кривая), которая в ИСО $S$ (в эфире) расширяется со скоростью $c$. Эта волна достигает центра отверстия в экране в момент времени $t^{\prime}=t$. Радиус волны в ИСО $S$ в момент $t$ составляет $c t$, а в ИСО $S^{\prime} c^{\prime} t$ или, что то же самое, $c^{\prime} t^{\prime}$. ИСО $S^{\prime}$ переместится при этом относительно ИСО $S$ на расстояние $V t$ вправо от начала координат ИСО $S$. Далее волна вырезается отверстием в экране и распространяется в виде отдельного сегмента волны.

Этот сегмент волны (свет) распространяется в ИСО $S$ под углом $\theta$ к положительной оси $x$ со скоростью $c$. $X$-компонента $c_{x}$ этой скорости в ИСО $S$ равна $V$ и направлена вдоль положительной оси $x$, а в ИСО $S^{\prime}$ $x$-компонента этой скорости, согласно преобразованию (13), равна нулю: $c_{x}^{\prime}=c_{x}-V=0$. То есть, таким образом, волна (свет) в ИСО $S^{\prime}$ распространяется под тем же самым углом, что и прожектор, т. е. вдоль оси $y^{\prime}$ в полном соответствии с формулой (1). Скорость света равна $c^{\prime}=c \sqrt{1-\frac{V^{2}}{c^{2}}}$.

Если же прожектор направить вдоль оси $x^{\prime}$, то свет (волна) также будет распространяться вдоль той же оси $x^{\prime}$, т.е. опять под тем же самым углом, что и прожектор. Скорость света будет равна $c_{ \pm}=c \pm V$, т.е. опять в полном соответствии с формулой (1). Здесь плюс соответствует свету, распространяющемуся влево, минус - вправо.

То есть, таким образом, мы показали, что скорость света в классической механике определяется именно формулой (1), а не (3) (или, что то же самое, формулой (4)). Свет в ИСО $S^{\prime}$ распространяется под тем же самым углом, что и прожектор. В ИСО $S$ направление распространения света и направление прожектора не совпадают.

\section{Список литературы}

[1] Малыкин Г.Б. // ЖТФ. 2007. Т. 77. Вып. 1. С. 138-140.

[2] Малыкин Г.Б. // УФН. 2009. Т. 179. С. 289. 